\title{
The Analysis and Research on Food Safety Risk Assessment Based on Data Mining
}

\author{
LiJuan $\mathrm{LI}^{1, \text { a }}$, YuanYing SHEN ${ }^{1}$, Zhiqiong $\mathrm{YUAN}^{1}$, Jie $\mathrm{LI}^{2}$ \\ ${ }^{1}$ School of Public Health, Dali University ,Dali, 671000,China \\ ${ }^{2}$ School of Mathematics and Computer Science ,Dali University, Dali 671003,China \\ alilijuan@126.com
}

\begin{abstract}
Keywords: Data Mining; Food Safety; Risk Assessment
\end{abstract}
\begin{abstract}
With the improvement of living standard, people more and more high to the requirement of food, the food safety problems emerge in endlessly, aroused the concern of the people for food safety and urgently need to establish a set of risk evaluation system, scientific and reasonable food for export food safety regulatory decision-making provide effective theoretical support. This paper proposes a data mining based food risk assessment and early warning method, set up a scientific and reasonable system of risk evaluation, risk evaluation results are obtained, and then through the evaluation result is multilayer multidimensional association rules mining, food and testing project early warning information, provides powerful guarantee for food safety.
\end{abstract}

\section{Introduction}

With the highlight of China's food safety problems and the public on the improvement of food safety requirements, and on our country's food safety management level of higher requirements are put forward. Based on the risk analysis framework of food safety risk management represents the latest achievements in modern science and technology the development direction of application in the food safety management, risk analysis framework to deal with and processing food safety problem has become the internationally accepted scientific principles and methods [1]. Food safety risk management not only refers to the government regulation, more contain the food safety chain on all kinds of stakeholders to participate. Food safety risk management have to do is beforehand control, is a source of food safety management, can play a fundamental role, realize the biggest benefit guarantee food safety. So as soon as possible to establish the risk analysis framework on the basis of food safety risk management system become the most important work of the current food safety management [2].

How to evaluate the food safety risk, and how to food or testing programs of early warning is a big problem in today's society. This paper proposes a data mining based food risk assessment and early warning method, first to establish a scientific and reasonable system of risk evaluation, risk evaluation results are obtained, and then through the evaluation result is multilayer multidimensional association rules mining, food and testing project early warning information, finally has carried on the design and implementation of the system. This paper deals with the food safety risk assessment system of the research can improve the quality of supervision, provides powerful guarantee for food safety.

\section{The basic technology of data mining}

Data mining is a long-term research and application of database technology is the inevitable result of the development of database technology, but a more advanced stage, it can not only a large number of historical data query, data can also be found in history unknown potential link [3]. In the face of the current state of large amounts of data, the association rule is an important branch of data mining, is the study of an advanced and intelligent data processing and analysis technology has become a hot. Through the mining of association rules, a lot of useful information can be hidden in the sea have the potential value of the data [4]. The goal of association rules is an effective method 
to extract the most interesting patterns. So far, many effective algorithms have been proposed for mining association rules mining algorithm is proposed, the algorithm of the most famous Agawal algorithm is based on a priori, but it is facing in the temporal and spatial scales of the challenge, therefore, many researchers are exploring a new mining method, concept and application of extended association rules.

Data mining refers to a large amount of data warehouses, the value is potentially important in the process of information reveals hidden, previously unknown, and data mining is a process of decision-making, which is based on artificial intelligence, machine learning, pattern recognition, statistics, database, visualization, highly automated analysis of enterprise data and make the inductive reasoning, digging out the potential model, to help the user to adjust marketing strategies to reduce the risk, make the right decision. From the process of data mining technology, as shown in figure 1.

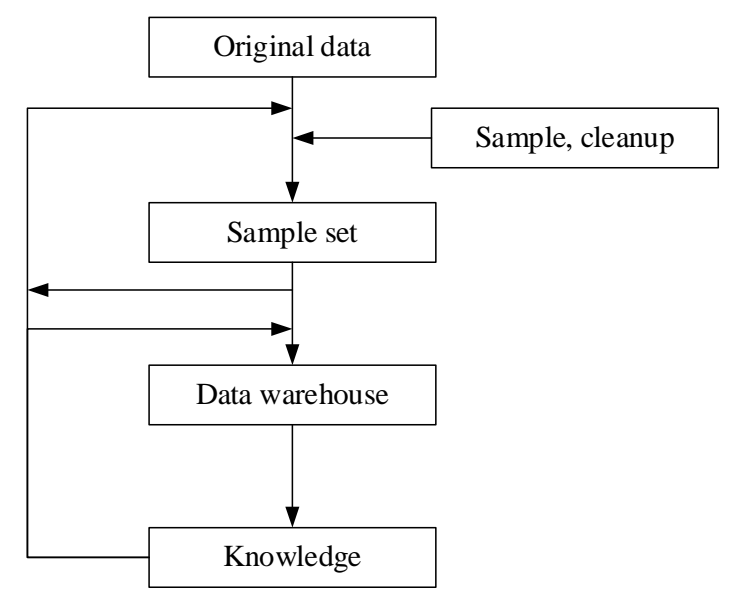

Figure 1.The basic process of data mining

After the data is collected, sampling and cleanup needs. Cleaning is the result of a sample data set. Data warehouse is an effective data storage, data mining is very useful. You can use different data mining algorithm. Sometimes, the final stage of the process need to be returned.

\section{The food safety risk assessment indicators}

The food safety risk assessment and early warning is the most pressing needs in recent years the food safety field, to early warning of a state object, the first thing to research paper analyzed the comprehensive evaluation of the state object. Risk evaluation system is an important link in the food safety risk assessment and early warning, to establish a reliable risk evaluation system not only provide scientific theory basis for food and the risk of early warning, also provide auxiliary decision making support for food safety supervision work [5]. Due to the risk evaluation index system is imperfect and experts is not convenience to risk evaluation index set, this paper set up a scientific and reasonable system of risk evaluation, first of all, a detailed analysis of the risk indicators of produce, perfect the risk assessment index system of risk evaluation index to identify, risk evaluation index to establish multidimensional data model, the information knowledge formalization, convenient experts on the level and set of weights. Then using comprehensive evaluation method to evaluate the risk of food, the food safety risk assessment index system is shown in figure 2. 


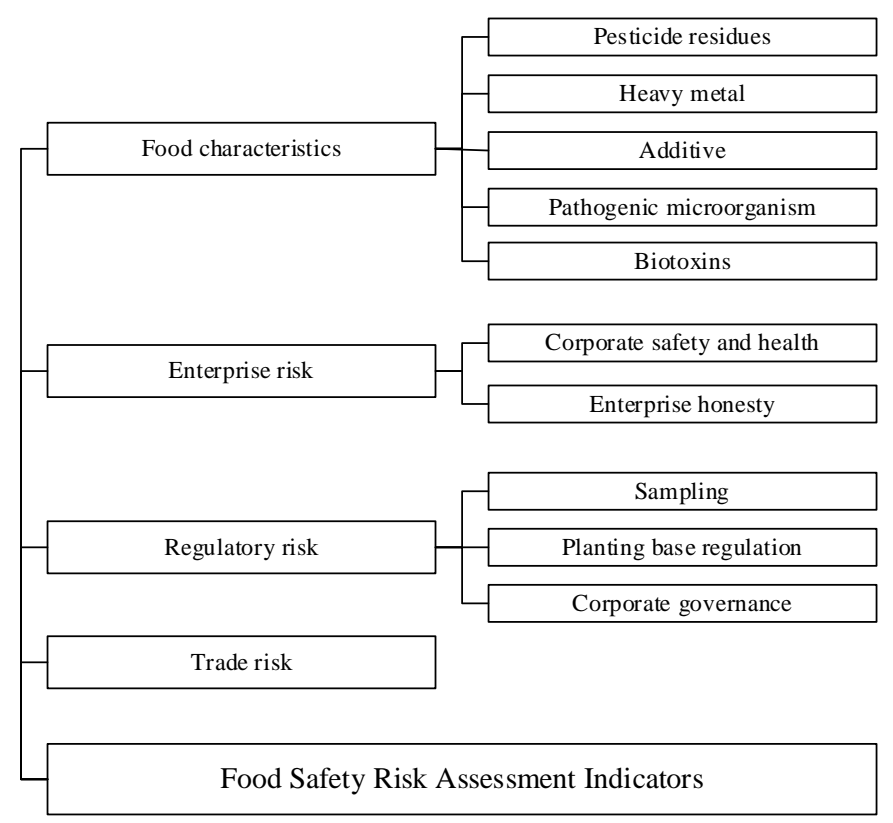

Figure 2.The food safety risk assessment index system

From the food safety risk assessment index system, it can be seen that the food safety risk assessment indicators are complex and diverse, and the food safety risk assessment index is hierarchical, different indicators by many indicators, son index number and the content is not the same, so to adopt hierarchical comprehensive evaluation method to evaluate risk indicators. In this paper, the construction of a multi-level comprehensive evaluation system, will again after subsystem separately carries on the comprehensive evaluation of the comprehensive evaluation system of the result as the lower input. According to the food safety risk assessment index system, constructs the comprehensive evaluation system level 4, level of the evaluation of the comprehensive evaluation factors for the probability of occurrence, detect unqualified data, social awareness, foreign technology level, the evaluation results can be used as a secondary evaluation factors in the comprehensive evaluation of the input value, and so on, to get food risk assessment level.

\section{The food safety risk assessment mechanism based on the technology of data mining}

Figure 3 depicts the food safety risk assessment mechanism based on data mining. Risk assessment system input interface, data input by the user interface, data mining module, risk factor of library, early warning function module and output interface. For managed automatic alarm, historical data and monitoring data through data input interface input to the system background, through the data mining module in risk factor in the library, data mining will demand information through the early warning function module, the early warning information output to the client interface; For managed artificial alarm: on-site management or security personnel through the inspection, found that the risk of production site information, through the interface to the client risk information input to the system background, through data mining module in risk factor in the library, data mining will demand information through the early warning function module, the risk information to the client output interface. 


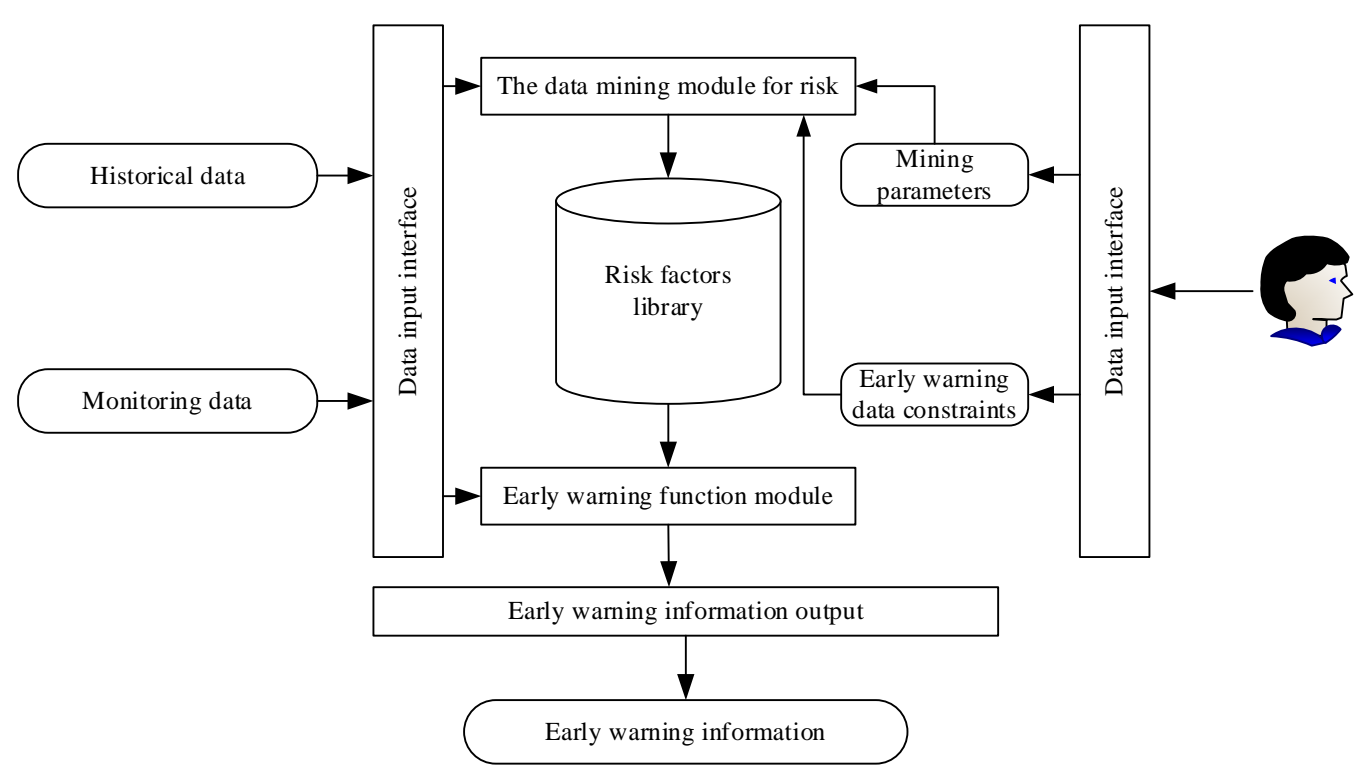

Figure 3. The food safety risk assessment mechanism based on data mining

According to the risk factors that can affect food inspection were analyzed, and using the Apriori algorithm for mining association rules. Because of food category, time, risk evaluation result is more value type data, and the Apriori algorithm for Boolean data, thus to historical evaluation data conversion.

\section{Conclusion}

Food safety problems occur frequently, the quality of food safety problems by the social people from all walks of life more and more seriously. How to evaluate the food safety risk, and how to food or testing programs of early warning is a big problem in today's society. At home and abroad about the food safety risk assessment and early warning method of theory study is less, there is no a set of universally accepted scientific and effective food safety risk assessment and early warning system, nor will the food safety risk assessment to better applied to the food safety risk management in actual application. This paper proposes a data mining based food risk assessment and early warning method, set up a scientific and reasonable risk evaluation system, optimize the working process of the food safety supervision, improve the quality of supervision and provides strong guarantee for food safety.

\section{Reference}

[1] R. Buyya, C.S. Yeo, and S. Venugopal: Future Generation computer systems, Vol. 25(2009) No.6, p. 599.

[2] G. Wei, A.V. Vasilakos, and Y. Zheng: The Journal of Supercomputing, Vol. 54(2011) No.2, p. 252.

[3] Y. Fang, and F. Wang, Ge J. Web Information Systems and Mining (Springer Berlin Heidelberg, 2010), p.271.

[4] Q. Zhang, L. Cheng, and R. Boutaba: Journal of internet services and applications, Vol. 1(2012) No.1, p.7

[5] M.D. Dikaiakos, D. Katsaros, and P. Mehra: Internet Computing, Vol. 13(2010) No.5, p. 10 